\title{
Biocompatible starch-halloysite hybrid: An efficient support for immobilizing Pd species and developing a heterogeneous catalyst for ligand and copper free coupling reactions
}

\author{
Samahe Sadjadi $^{1}$, Masoumeh Malmir ${ }^{2}$, Majid M.Heravi $^{2}$, Fatemeh Ghoreyshi Kahangi $^{3}$
}

1- Gas Conversion Department, Faculty of Petrochemicals, Iran Polymer and Petrochemicals Institute, PO Box 14975-112, Tehran, Ira

2- Department of Chemistry, School of Science, Alzahra University, PO Box 1993891176, Vanak, Tehran, Iran

3- Department of Chemistry, University Campus 2, University of Gilan, Rasht, Iran

\section{Abstract}

Combining the exceptional features of halloysite and starch, a unique hybrid was synthesized based on conjugation of amine-functionalized starch with $\mathrm{Cl}$ halloysite nanoclay followed by coordination of Pd (II) acetate. The resultant hybrid catalyst, Pd@Hal-SA, was used as a heterogeneous catalyst for promoting copper and ligand-free coupling reactions under mild reaction condition. The catalyst exhibited high catalytic activity and recyclability (up to 10 reaction runs) with slight loss of the catalytic activity that stemmed from slight Pd leaching. The comparison of the catalytic activity of Pd-Hal, Pd-SA and Pd@Hal-SA confirmed the superior performance of the latter, indicating the contribution of two components, SA and Hal to the catalysis. Moreover, the catalytic activity of Pd@Hal-SA was higher than that of Pd@Hal + SA, implying that the use of hybrid system was more efficient that use of individual components in a separated form, due to the synergism between SA and Hal.

Keywords: Halloysite nanotube, Starch, Pd nanoparticles, Heterogeneous catalyst 